\title{
All-trans retinoic acid-encapsulated, CD20 antibody-conjugated poly(lactic-co-glycolic acid) nanoparticles effectively target and eliminate melanoma-initiating cells in vitro
}

This article was published in the following Dove Press journal:

OncoTargets and Therapy

\section{Xingyu Chen ${ }^{1,2}$ \\ Zhiyuan Zhang ${ }^{3}$ \\ Shengfeng Yang ${ }^{4}$ \\ Hairong Chen ${ }^{2}$ \\ Dan Wang ${ }^{5}$ \\ Jun $\mathrm{Li}^{6}$}

'Department of Dermatology and Venerology, Shandong University School of Medicine, Jinan, Shandong, 250000, China; ${ }^{2}$ Department of Dermatology, Qingdao Municipal Hospital, Qingdao, Shandong 2660II, China; ${ }^{3}$ Department of Neurosurgery, Liaocheng People's Hospital, Liaocheng, Shandong 252000, China; ${ }^{4}$ Department of Medical Oncology, Qingdao Center Hospital, Qingdao, Shandong 2660II, China; ${ }^{5}$ Department of Ultrasound, Liaocheng People's Hospital, Liaocheng, Shandong 252000, China; ${ }^{6}$ College of Pharmacy, Liaocheng University, Liaocheng, Shandong 252000, China

Correspondence: Xingyu Chen Department of Dermatology, Qingdao Municipal Hospital, No. I JiaoZhou Road, Qingdao, Shandong 2660I I, China

Tel +865328285 2008

Fax +86 53282836421

Email chenxingyu2008@I63.com

Jun $\mathrm{Li}$

Department of Pharmacy, College of Pharmacy, Liaocheng University,

No. I Hu'nan Road, Liaocheng,

Shandong 252000, China

Tel +86 6358239133

Fax +86 6358239680

Email think.a@I63.com
Purpose: Melanoma, which is initiated from melanocytes, is the most fatal type of skin cancer. Melanoma-initiating cells significantly contribute to the initiation, metastasis, and recurrence of melanoma, and CD20 is a marker of melanoma-initiating cells. All-trans retinoic acid (ATRA) has been demonstrated to induce differentiation, inhibit proliferation, and promote the apoptosis of cancer cells and cancer-initiating cells (CICs). However, there has been no report on ATRA activity against melanoma-initiating cells. In this study, we examined the activity of ATRA against melanoma-initiating cells and developed ATRA-encapsulated poly(lactic-co-glycolic acid) (PLGA) nanoparticles, which were conjugated with a CD20 antibody (ATRA-PNP-CD20) for targeted delivery of ATRA to $\mathrm{CD} 20^{+}$melanoma-initiating cells.

Materials and methods: The effects of ATRA and ATRA-PNP-CD20 against melanomainitiating cells were investigated using a cytotoxicity assay, tumorsphere formation assay, and flow cytometry.

Results: ATRA-PNP-CD20 had a size of $126.9 \mathrm{~nm}$ and a negative zeta potential. The drugloading capacity of ATRA-PNP-CD20 was $8.7 \%$, and ATRA-PNP-CD20 displayed a sustained release of ATRA for 144 hours. The results showed that ATRA-PNP-CD20 could effectively and specifically deliver ATRA to CD20+ melanoma-initiating cells, achieving superior inhibitory effects against $\mathrm{CD} 20^{+}$melanoma-initiating cells compared with those of free ATRA and nontargeted nanoparticles. To the best of our knowledge, we report for the first time a potent activity of ATRA against CD20 ${ }^{+}$melanoma-initiating cells, targeted drug delivery of ATRA via nanoparticles to melanoma-initiating cells, and the achievement of a superior inhibitory effect against melanoma-initiating cells by using a CD20 antibody.

Conclusion: ATRA-PNP-CD20 represents a promising tool for eliminating melanoma-initiating cells and shows a potential for the therapy of melanoma.

Keywords: melanoma, cancer-initiating cells, nanoparticles, CD20, antibody

\section{Introduction}

Melanoma, which is initiated from melanocytes, represents an aggressive and fatal cancer. The US statistics indicate that the rates of melanoma in the US have been on the rise in the past 30 years. ${ }^{1}$ For human beings, melanoma remains a significant mortality burden. Although it only accounts for $\sim 1 \%$ of skin cancer, melanoma is resistant to many chemotherapeutics and represents the most fatal type of skin cancer. ${ }^{2}$ The number of deaths has been reported to be 2.7 per 100,000 people per year in USA. ${ }^{1}$ Therefore, the development of a therapy for melanoma is an urgent need for human health. 
Although great achievements have been made in melanoma therapy, a treatment failure and decrease in survival are often encountered because of recurrence, metastasis, and multidrug resistance of melanoma, ${ }^{3,4}$ which are considered to be attributable to melanoma-initiating cells. ${ }^{3-6}$ Therefore, the elimination of melanoma-initiating cells may contribute to the cure of melanoma. CD20, an activated glycosylated phosphoprotein, which is expressed on B cells, is considered a marker for melanoma-initiating cells..$^{5-9}$ Fang et $\mathrm{al}^{8}$ showed that $\mathrm{CD} 20^{+}$melanoma cells are more aggressive than their counterparts, CD20- melanoma cells, as reflected by their higher proliferative, clonogenic, and tumorigenic abilities. In addition, CD $20^{+}$melanoma cells can rapidly form tumorspheres and differentiate into different cell types. ${ }^{8}$ It is noteworthy that the elimination of CD $20^{+}$melanoma cells could permanently eliminate melanoma. ${ }^{9}$ On the contrary, the elimination of melanoma could not be achieved by eliminating other melanoma subpopulations. ${ }^{9}$ In several patients with stage IV metastatic melanoma, rituximab, an anti-CD20 antibody, exhibited a significant therapeutic effect against melanoma. ${ }^{10}$ Taken together, the $\mathrm{CD} 20^{+}$melanomainitiating cell subpopulation is crucial for the initiation, metastasis, and recurrence of melanoma. Targeted eradication of this subpopulation should be an effective treatment for melanoma. ${ }^{9,10}$

All-trans retinoic acid (ATRA), an active metabolite of vitamin A, belonging to the retinoid family, is a promising drug, shown to cause differentiation, inhibition of proliferation, and apoptosis of cancer cells in various cancers. ${ }^{11,12}$ An ATRA-based differentiation therapy is regarded as a significant advance in cancer therapy. ATRA has become the first-choice drug for the therapy of acute promyelocytic leukemia (APL) ${ }^{11}$ and has also been demonstrated to be effective in treating APL as an adjuvant. ${ }^{12}$ Strikingly, ATRA has shown a therapeutic potential against cancerinitiating cells (CICs) in several cancers, such as breast cancer, glioblastoma multiforme, and sarcoma. ${ }^{13-15}$ In these studies, ATRA significantly inhibited the self-renewal and proliferative abilities and promoted the apoptosis of CICs, suggesting that the compound represents a promising drug against CICs. ${ }^{13-15}$ ATRA has also been reported to exert promising therapeutic effects against melanoma cells via different mechanisms, including mitochondrial dysfunction, an altered cell cycle, induction of apoptosis, and modulation of carbohydrate sulfotransferase $10 .{ }^{16,17}$ However, there have been no studies reporting the therapeutic effect of ATRA on melanoma-initiating cells. ${ }^{18-20}$

Meanwhile, the aqueous solubility of ATRA is poor, resulting in its low bioavailability and poor therapeutic effects in vivo. ${ }^{13}$ It is known that nanoparticle-based strategies can remarkably improve the bioavailability and therapeutic index of conventional therapeutics by improving the solubility of poorly soluble drugs and providing targeted delivery of drugs. ${ }^{21-23}$ Several studies have developed ATRA-loaded nanoparticles to facilitate the preclinical application of ATRA in cancer therapy. ${ }^{13,24}$ In these studies, the solubility and bioavailability of ATRA remarkably increased, and ATRAloaded nanoparticles exhibited a superior therapeutic efficacy against cancer compared with that of ATRA.

Nanoparticles made of biodegradable polymers represent a superior candidate drug delivery system. Their advantages include controlled and sustained release, high drug loading, and superior stability. ${ }^{25,26}$ Poly(lactic-co-glycolic acid) (PLGA) nanoparticles are one of the most used types of nanoparticles made of biodegradable polymers because of their superior biocompatibility and flexibility in modulating drug release. ${ }^{25,26}$ Commonly, poly(ethylene glycol) (PEG) chains are incorporated as copolymers in nanoparticles to increase their hydrophilicity, modification flexibility, and circulation time. ${ }^{23-26}$ For targeted delivery of chemotherapy drugs to cancer cells, considerable attention has been paid to antibody-conjugated nanoparticles. ${ }^{27-29}$ It is well known that antibody-targeted nanoparticles improve the therapeutic effect of chemotherapy in various cancers. ${ }^{25,26}$ Since CD20 is a marker of melanoma-initiating cells, we hypothesized that a CD20 antibody could be used for targeted delivery of ATRA-loaded nanoparticles to melanoma-initiating cells. We have previously used nanoparticles targeting CD20 to deliver various drugs, including vincristine and salinomycin, to $\mathrm{CD} 20^{+}$melanoma stem cells for targeted chemotherapy. ${ }^{30,31}$

The objective of the present study was to target melanomainitiating cells by constructing ATRA-encapsulated, CD20 antibody-conjugated PLGA nanoparticles.

\section{Materials and methods Materials}

PLGA (20,000 Da)-PEG (5,000 Da)-maleimide (MAL), coumarin 6, polyvinyl alcohol (30-70 kDa), 2-iminothiolane (Traut's reagent), ATRA, and organic reagents were all purchased from Sigma-Aldrich Co. (St Louis, MO, USA). The CD20 microbead kit was provided by Miltenyi Biotec (Shanghai, China). R\&D Systems, Inc. (Minneapolis, MN, USA) provided a recombinant antihuman CD20 Alexa Fluor ${ }^{\circledR}$ 488-conjugated antibody and a recombinant mouse antihuman CD20 monoclonal antibody. The CD20 Fab' from the recombinant mouse antihuman $\mathrm{CD} 20$ monoclonal antibody was obtained according to our previous protocol. ${ }^{26}$ A Pierce 
bicinchoninic acid protein assay kit, DMEM, FBS, B27 supplement, EGF, basic fibroblast growth factor (bFGF), and insulin-transferrin-selenium (ITS) were purchased from Thermo Fisher Scientific (Waltham, MA, USA).

\section{Culture of melanoma cells}

Two human melanoma cell lines, A375 and WM266-4, were obtained from the American Type Culture Collection (ATCC, Manassas, VA, USA) and maintained at $37^{\circ} \mathrm{C}$ in DMEM supplemented with 10\% FBS, 4-(2-hydroxyethyl)1-piperazineethanesulfonic acid $(25 \mathrm{mM})$, streptomycin $(100 \mu \mathrm{g} / \mathrm{mL})$, and penicillin $(100 \mathrm{U} / \mathrm{mL})$.

\section{CD20 expression in melanoma cell lines}

Flow cytometry was carried out to analyze the expression of CD20 in the cell lines. In brief, dissociated melanoma cells were treated with the antihuman CD20 Alexa Fluor ${ }^{\circledR} 488$ conjugated antibody at $1 \mu \mathrm{g} / \mathrm{mL}$ for 0.5 hours at $4^{\circ} \mathrm{C}$ and then washed three times with PBS. The washed cells were suspended in PBS, and an FACS Calibur flow cytometer (flow cytometry; BD, Franklin Lakes, NJ, USA) was used to analyze the proportion of positively stained cells.

\section{Magnetic bead-based cell sorting}

Separation of a CD20+ cell subpopulation from melanoma cells was performed according to the protocol provided by Miltenyi Biotec with the CD20 microbead kit, and the FACS Calibur FCM was used to analyze the proportion of positively stained cells, as described earlier.

\section{Formation of tumorspheres by melanoma cells}

The formation of tumorspheres was used to evaluate the selfrenewal ability of melanoma-initiating cells. In brief, melanoma cells were suspended in a stem cell medium and plated at a density of 5,000 cells/well in Corning ${ }^{\circledR}$ ultralow adherent six-well dishes (Corning Incorporated, Corning, NY, USA). The stem cell medium contained DMEM/F12, B27 supplement $(1 \times), \operatorname{ITS}(1 \times), E G F$, and bFGF (both at a concentration of 20 $\mathrm{ng} / \mathrm{mL}$ ). The cells were cultured in the stem cell medium for 7 days, and then tumorspheres were counted under a conventional microscope. For second passage tumorspheres, those of the first passage were washed with PBS, dissociated with a cell dissociation reagent (StemPro ${ }^{\circledR}$ Accutase $^{\circledR}$; Thermo Fisher Scientific, Waltham, MA, USA), and then propagated.

\section{Tumorigenicity of melanoma cells in mice}

An in vivo tumorigenicity assay was carried out by inoculation of melanoma cells into BALB/c nude mice (4-5 weeks old, males, $\sim 20 \mathrm{~g})$. The mice were purchased from the Experimental Animal Center of Shandong (Jinan, China). All procedures were approved by and performed in accordance with the guidelines of the Committee on Animals of Liaocheng University (Shandong, China). In brief, CD20 ${ }^{+}$and CD20- melanoma cells were isolated using magnetic beads, and then $2 \times 10^{3}-1 \times 10^{6}$ cells were mixed with BD Matrigel ${ }^{\mathrm{TM}}$, BD Biosciences, San Jose, CA, USA. The mixture of the cells and gel was implanted subcutaneously into the mice. The tumor formation was recorded during an observation period of 15 weeks.

\section{Preparation of ATRA-encapsulated PLGA nanoparticles}

ATRA-encapsulated PLGA nanoparticles were produced by an emulsion/solvent evaporation approach. PLGA-PEGMAL and ATRA (10:1, w/w) were dissolved in dichloromethane $(2 \mathrm{~mL})$, and the solution was then added to a $1.5 \%$ sodium cholate solution $(5 \mathrm{~mL})$. A probe sonicator was used to sonicate the resultant solution (1 minute, $180 \mathrm{~W}$ ). The resultant emulsion was added to a $0.5 \%$ sodium cholate solution $(20 \mathrm{~mL})$, and then dichloromethane was removed by evaporation overnight. Non-loaded ATRA was removed by ultrafiltration through an Amicon Ultra-15 centrifugal filter device (EMD Millipore, Billerica, MA, USA) with a nominal molecular weight cutoff of $100 \mathrm{kDa}$. Blank nanoparticles were produced in the same way, without the addition of ATRA. To obtain coumarin 6-labeled nanoparticles, $0.1 \%$ $(\mathrm{w} / \mathrm{w})$ coumarin 6 was added to an initial mixture of the polymers and the drug. Furthermore, CD20 Fab' was thiolated with 2-iminothiolane (molar ratio of Fab' to 2-iminothiolane was 1:100). The thiolated CD20 Fab' was conjugated with nanoparticles (mass ratio of $\mathrm{Fab}^{\prime} /$ nanoparticles was 1/20) for 6 hours at room temperature, and the unconjugated $\mathrm{Fab}^{\prime}$ was removed using an Amicon Ultra-15 centrifugal filter device with a nominal molecular weight cutoff of $100 \mathrm{kDa}$.

The following abbreviations are used to designate nanoparticles: ATRA-PNP, ATRA-encapsulated PLGA nanoparticles; ATRA-PNP-CD20, ATRA-encapsulated, CD20 antibody-conjugated PLGA nanoparticles; and PNP-CD20, blank CD20 antibody-conjugated PLGA nanoparticles.

\section{Antibody conjugation efficiency of nanoparticles}

Ultrafiltration of nanoparticles was used to evaluate their antibody conjugation efficiency. Briefly, the antibody was incubated with nanoparticles, and then the mixture was centrifuged through an Amicon centrifugal filter (nominal molecular weight cutoff: $100 \mathrm{kDa}$ ) to remove the unconjugated antibody, which was measured using the Pierce protein assay reagent kit (Thermo Fisher 
Scientific, Inc., Waltham, MA, USA). The antibody conjugation efficiency of nanoparticles was evaluated using the following equation: $\left(M_{t}-M_{u}\right) / M_{t}$, where $M_{t}$ is the total mass of the antibody, and $\mathrm{M}_{\mathrm{u}}$ is the mass of the unconjugated antibody.

\section{Size, zeta potential, morphology, and drug-loading capacity of PLGA nanoparticles}

The particle size and zeta potential were evaluated using a Zetasizer Nano ZS90 (Malvern Instruments, Malvern, UK) after diluting $100 \mu \mathrm{L}$ of nanoparticles in $1.9 \mathrm{~mL}$ of distilled water. The ATRA encapsulation efficiency and loading capacity of PLGA nanoparticles were determined by reversed-phase HPLC on an Agilent system (Agilent Technologies, Santa Clara, CA, USA) with an ODS column (Diamonsil $^{\circledR}, 250 \times 4.6 \mathrm{~mm}, 5 \mu \mathrm{m}$ ). The mobile phase was acetic acid/acetonitrile/water $(0.5 / 95 / 4.5, \mathrm{v} / \mathrm{v} / \mathrm{v})$, and the flow rate was $1.0 \mathrm{~mL} / \mathrm{min}$. The detection wavelength of ATRA, using an ultraviolet detector, was set at $350 \mathrm{~nm}$. The loading of coumarin 6 into nanoparticles was evaluated using a standard coumarin 6 calibration curve.

\section{ATRA release from PLGA nanoparticles}

PLGA nanoparticles $(0.5 \mathrm{mg} / \mathrm{mL})$ were suspended in PBS or PBS with $10 \% \mathrm{FBS}$ in a centrifuge tube and then gently shaken on an orbital shaker at $37^{\circ} \mathrm{C}$ at $100 \mathrm{rpm}$. The tubes were centrifuged (20,000 rpm for 30 minutes) at designated time points during a 120-hour drug release period. Then, the supernatant was removed and measured by HPLC as described earlier. The cumulative ATRA release rate from nanoparticles was calculated using the following formula: $\left(M_{i} / M_{t}\right) \times 100 \%$, where $M_{i}$ is the mass of released ATRA, and $\mathrm{M}_{\mathrm{t}}$ is the total amount of ATRA.

\section{In vitro targeting of fluorescent nanoparticles to melanoma cells}

We used flow cytometry, as described earlier, to examine in vitro targeting of fluorescent nanoparticles. Briefly, melanoma cells $\left(5 \times 10^{5}\right.$ cells/well $)$ were cultured on 12 -well cell culture plates overnight at $37^{\circ} \mathrm{C}$. After that, the medium was replaced with a fresh medium containing coumarin 6-labeled nanoparticles ( $25 \mathrm{ng} / \mathrm{mL}$ coumarin 6). After 2-hour incubation, the cells were washed three times with PBS to remove unbound nanoparticles and dissociated into single cells with trypsin. The cells were suspended in PBS and analyzed using the FACS Calibur FCM.

\section{Cytotoxic effects of nanoparticles on melanoma cell lines}

The cytotoxic effects of nanoparticles were examined using the cell counting kit-8 (CCK-8) assay, as described by the manufacturer. Briefly, melanoma cells were washed, trypsinized, and seeded at a density of $3 \times 10^{3}$ cells/well in 96-well cell culture plates. After overnight incubation, the medium was replaced with a fresh medium containing ATRA or nanoparticles at a series of concentrations. After 72-hour treatment, the medium was replaced with a fresh medium, and cell viability was examined by the CCK- 8 assay with a microplate reader (Multiskan MK3). The data were processed using GraphPad Prism (GraphPad Software, Inc., La Jolla, CA, USA) to calculate the half-maximal inhibitory concentration $\left(\mathrm{IC}_{50}\right)$ values.

\section{Impact of nanoparticles on the proportion of melanoma-initiating cells in melanoma cell population}

Tumorsphere formation and the proportion of $\mathrm{CD} 20^{+}$cells were examined to evaluate the impact of nanoparticles on the proportion of melanoma-initiating cells in the melanoma cell population. In brief, melanoma cells were washed, trypsinized into single cells, and seeded at a density of $5 \times 10^{4}$ cells/well in 12-well cell culture plates. After overnight incubation, the cells were washed with PBS and then treated with a fresh medium containing nanoparticles (at a concentration equivalent to $15 \mu \mathrm{g} / \mathrm{mL}$ ATRA). Twenty-four hours later, the medium was aspirated, and the cells were washed, followed by the addition of a fresh medium and incubation for 72 hours. Then, the cells were washed and trypsinized, and the formation of tumorspheres was evaluated as described earlier. Alternatively, flow cytometry was used to measure the percentage of $\mathrm{CD} 20^{+}$cells in the population of trypsinized cells.

\section{Statistical analyses}

Differences between two groups were evaluated with the Student's non-paired $t$-test, and differences among three or more groups were calculated by one-way ANOVA. $P$-values of $<0.05$ were considered statistically significant. All data were presented as mean $\pm \mathrm{SD}$, unless otherwise stated.

\section{Results}

\section{Fabrication and characterization of PLGA nanoparticles}

ATRA-PNP nanoparticles were fabricated using an emulsion/ solvent evaporation approach. After the preparation of 
Table I The size and other characteristics of PLGA nanoparticles

\begin{tabular}{l|l|l|l|l|l}
\hline Nanoparticles & Size $(\mathbf{n m})$ & $\begin{array}{l}\text { Zeta potential } \\
(\mathbf{m V})\end{array}$ & PDI & $\begin{array}{l}\text { Drug } \\
\text { loading (\%) }\end{array}$ & $\begin{array}{l}\text { EE (\%) } \\
\text { ATRA-PNP }\end{array}$ \\
ATRA-PNP-CD20 & $118.3 \pm 16.3$ & $-15.3 \pm 6.8$ & $0.15 \pm 0.07$ & $9.7 \pm 2.6$ & $79.9 \pm 9.2$ \\
PNP-CD20 & $126.9 \pm 15.9$ & $-18.6 \pm 7.3$ & $0.13 \pm 0.03$ & $8.7 \pm 3.5$ & $78.6 \pm 7.6$ \\
\hline
\end{tabular}

Notes: Data are expressed as mean \pm SD $(n=4)$. ATRA-PNP, ATRA-encapsulated PLGA nanoparticles; ATRA-PNP-CD20, ATRA-encapsulated PLGA nanoparticles conjugated with CD20 antibodies; PNP-CD20, blank PLGA nanoparticles conjugated with CD20 antibodies.

Abbreviations: ATRA, all-trans retinoic acid; ATRA-PNP, ATRA-encapsulated PLGA nanoparticles; ATRA-PNP-CD20, ATRA-encapsulated PLGA nanoparticles conjugated with CD20 antibodies; PNP-CD20, blank PLGA nanoparticles conjugated with CD20 antibodies; EE, encapsulation efficacy; PDI, polydispersity index; PLGA, poly(lactic-co-glycolic acid).

ATRA-PNP, the thiolated antibody was linked to PLGA via the reaction of the sulfhydryl group on the antibody and the MAL group of the PLGA nanoparticles. The sizes, zeta potentials, and drug-loading capacities of the nanoparticles are summarized in Table 1. ATRA-PNP, not conjugated with the antibody, showed a small size of $118.3 \mathrm{~nm}$. However, after antibody conjugation, the sizes of nanoparticles were somewhat larger (126.9 and $129.1 \mathrm{~nm}$ for ATRA-PNP-CD20 and PNP-CD20, respectively). The zeta potentials of all the nanoparticles were negative and approximately $-15 \mathrm{mV}$. The drug-loading capacities (the amount of entrapped drug divided by the total nanoparticle weight) of ATRA-PNP and ATRA-PNP-CD20 were approximately $9.7 \%$ and $8.7 \%$, respectively. The encapsulation efficiencies (the percentage of drug successfully entrapped into the nanoparticle) of ATRA-PNP and ATRA-PNP-CD20 were approximately $79.9 \%$ and $78.6 \%$, respectively. The antibody conjugation efficiency of ATRAPNP-CD20 was $\sim 15 \%$. The ATRA release data, as shown in Figure 1, indicated that all the nanoparticles displayed a sustained release of ATRA during the 144-hour period. The ATRA release from the nanoparticles was markedly higher in PBS $+10 \%$ FBS than in PBS $(P<0.05)$, suggesting that serum could destabilize the structure of nanoparticles and facilitate the ATRA release.

\section{The CD20+ subpopulation of melanoma cells showed properties of melanoma- initiating cells}

CD20 microbead-based cell sorting was used to isolate CD20 cells from the population of melanoma cells, and the results showed that $>99 \%$ of the isolated cells were CD $20^{+}$cells. In contrast, the original melanoma cell lines showed only a low percentage of $\mathrm{CD} 20^{+}$cells $(\sim 7 \%)$. The tumorsphere formation assay was used to identify melanoma-initiating cells. Figure 2A shows that the number of tumorspheres generated by $\mathrm{CD} 20^{+} \mathrm{A} 375$ cells was remarkably higher than that generated by $\mathrm{CD} 20^{-} \mathrm{A} 375$ cells (first passage: $P<0.01$; second passage: $P<0.001)$. In WM266-4 cells, similar results were achieved (first passage: $P<0.01$; second passage: $P<0.001$; Figure 2B). In addition, $\mathrm{CD} 20^{+}$cells showed a higher capability for melanoma formation in nude mice than did $\mathrm{CD}^{-} 0^{-}$cells (Figure 2C and D). Compared with those induced by $\mathrm{CD} 20^{-}$cells, the tumor volumes were remarkably higher after day 22 for CD20 ${ }^{+}$A375 and WM266-4 cells $(P<0.05)$. On day 34, the tumor volume induced by $\mathrm{CD} 20^{+}$ A375 cells was $300 \mathrm{~mm}^{3}$, and that induced by CD20- A375 cells was $120 \mathrm{~mm}^{3}$ ( $P<0.001$; Figure 2C). In WM266-4 cells, similar results were achieved (Figure 2D). On day 34, the tumor volume induced by CD20+ WM266-4 cells was $986 \mathrm{~mm}^{3}$, and that induced by CD20- WM266-4 cells was $209 \mathrm{~mm}^{3}(P<0.001)$.

Subsequently, we evaluated tumorigenicity by using different numbers of melanoma cells in mice (Table 2). Strikingly, a 100\% incidence of tumors (10/10) occurred

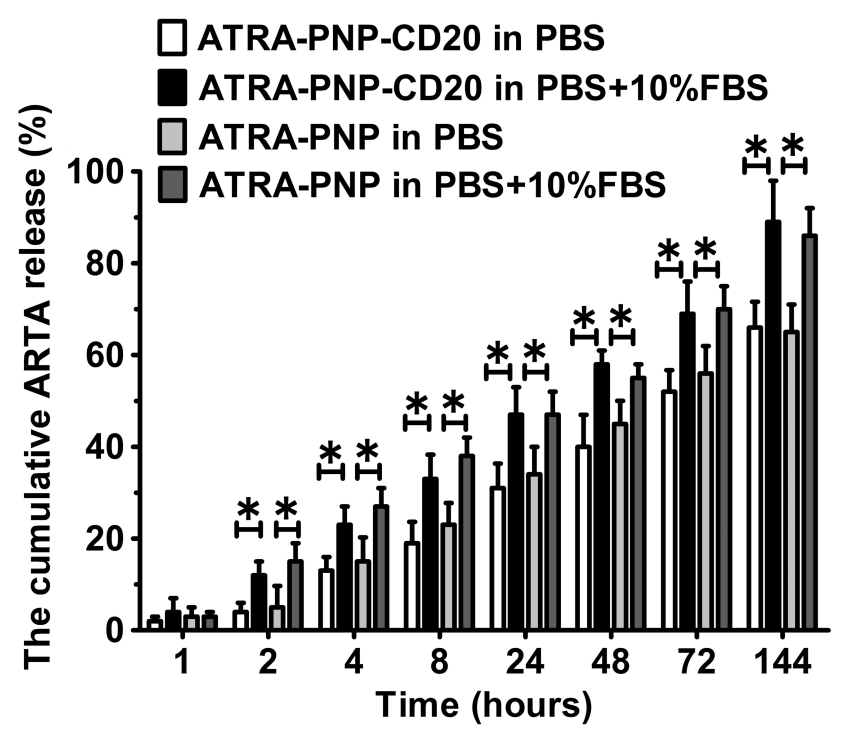

Figure I ATRA release from nanoparticles.

Notes: The release media were PBS and PBS +10\% FBS. The difference between two groups was detected by a non-paired Student's $t$-test. $* P<0.05$. Data are presented as mean $\pm S D(n=3)$. ATRA-PNP, ATRA-encapsulated PLGA nanoparticles; ATRAPNP-CD20, ATRA-encapsulated PLGA nanoparticles conjugated with CD20 antibodies.

Abbreviations: ATRA, all-trans retinoic acid; PLGA, poly(lactic-co-glycolic acid). 


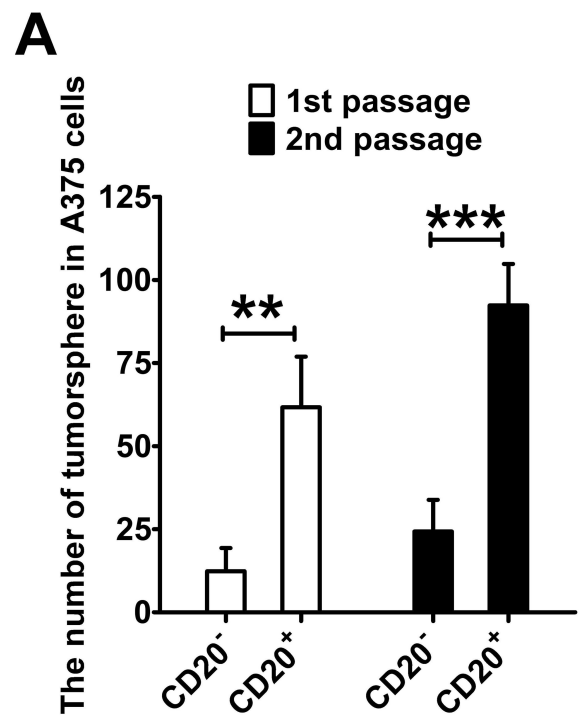

B
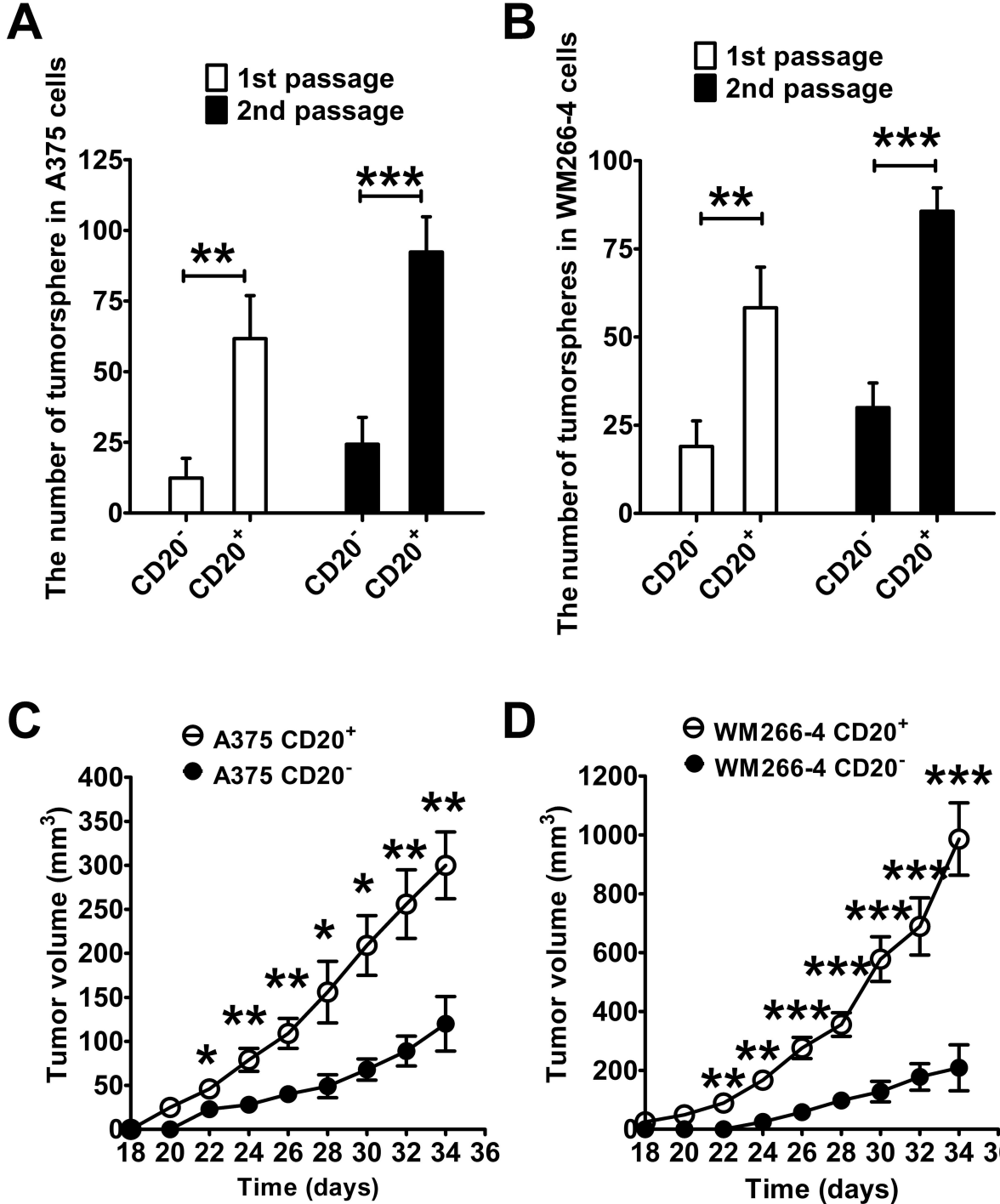

D

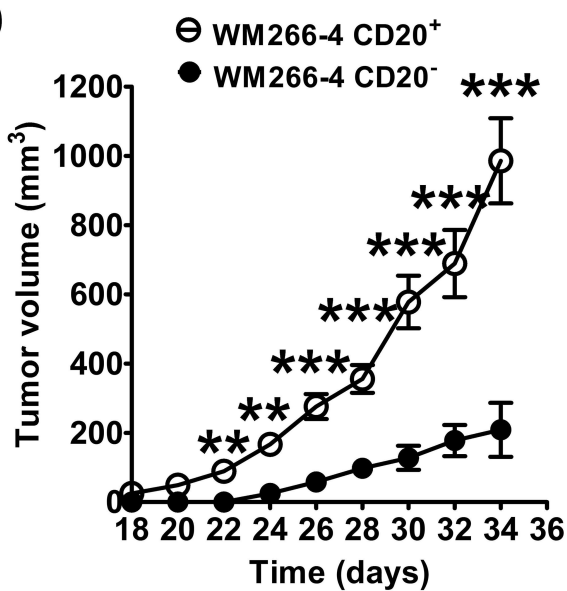

Figure $2 \mathrm{CD}_{2} \mathrm{O}^{+}$melanoma cells have properties of melanoma-initiating cells, as demonstrated by the tumorsphere formation and tumor growth in nude mice. Notes: (A, B) Tumorsphere formation in a serum-free medium. (C, D) A growth curve of melanoma in nude mice injected with $2 \times 10^{5}$ melanoma cells. The difference between two groups was detected by a non-paired Student's $t$-test. $* P<0.05 ; * * P<0.01 ; * * * P<0.00$ I. Data are presented as mean $\pm S D(n=6)$.

in the mice inoculated with $\geq 1 \times 10^{4} \mathrm{CD} 20^{+} \mathrm{A} 375$ cells. On the contrary, only a $60 \%$ incidence of tumors $(6 / 10)$ was found in the mice inoculated with $\mathrm{CD} 20^{-} \mathrm{A} 375$ cells, even though the cell number was $1 \times 10^{6}$, indicating that $\mathrm{CD} 20^{+}$ A375 cells had a significantly higher tumorigenic potential than did CD20- A375 cells. Similarly, CD20+ WM266-4 cells had a significantly higher tumorigenic ability than did

Table 2 The in vivo tumorigenicity of melanoma cells in nude mice

\begin{tabular}{l|l|l|l|l|l|l}
\hline Types & $\mathbf{I} \times 10^{6}$ & $\mathbf{I} \times 10^{5}$ & $\mathbf{2} \times \mathbf{1 0 ^ { 4 }}$ & $\mathbf{I} \times \mathbf{1 0 ^ { 4 }}$ & $\mathbf{5} \times \mathbf{1 0 ^ { 3 }}$ & $\mathbf{2 \times 1 0 ^ { 3 }}$ \\
\hline CD20- A375 & $6 / 10$ & $3 / 10$ & $2 / 10$ & $1 / 10$ & $0 / 10$ & $0 / 10$ \\
CD20 A375 & $10 / 10$ & $10 / 10$ & $10 / 10$ & $10 / 10$ & $8 / 10$ & $6 / 10$ \\
CD20- WM266-4 & $8 / 10$ & $6 / 10$ & $2 / 10$ & $1 / 10$ & $0 / 10$ & $0 / 10$ \\
CD20+ WM266-4 & $10 / 10$ & $10 / 10$ & $10 / 10$ & $10 / 10$ & $8 / 10$ & $4 / 10$ \\
\hline
\end{tabular}

Notes: $\mathrm{CD}_{20} \mathrm{O}^{+}$or $\mathrm{CD}^{-} \mathrm{O}^{-}$melanoma cells were collected, mixed with matrigel, and implanted subcutaneously into BALB/c nude mice. The tumor formation was recorded during the observation period of 15 weeks.
CD20- WM266-4 cells. CD20+ WM266-4 cells produced tumors in mice with a $100 \%$ incidence at a cell number of $\geq 1 \times 10^{4}$ cells, whereas $1 \times 10^{4} \mathrm{CD} 20^{-}$WM266- 4 cells only produced tumors with a $10 \%$ incidence $(1 / 10)$. In summary, the tumorigenicity of $\mathrm{CD} 20^{+}$melanoma cells was remarkably higher than that of $\mathrm{CD} 20^{-}$melanoma cells.

\section{In vitro targeting of fluorescent nanoparticles to melanoma cells}

Coumarin 6 is a green fluorescent tracer that has been widely used to measure the uptake of nanoparticles. In this study, coumarin 6 was used to evaluate the in vitro targeting of fluorescent nanoparticles to cancer cells. As shown in Figure 3A, the uptake of coumarin 6-labeled ATRA-PNP$\mathrm{CD} 20$ by $\mathrm{CD} 20^{+} \mathrm{A} 375$ cells was prominently higher than that of coumarin 6-labeled ATRA-PNP and free coumarin 6 $(P<0.01)$. However, CD20- A375 cells showed a similar 

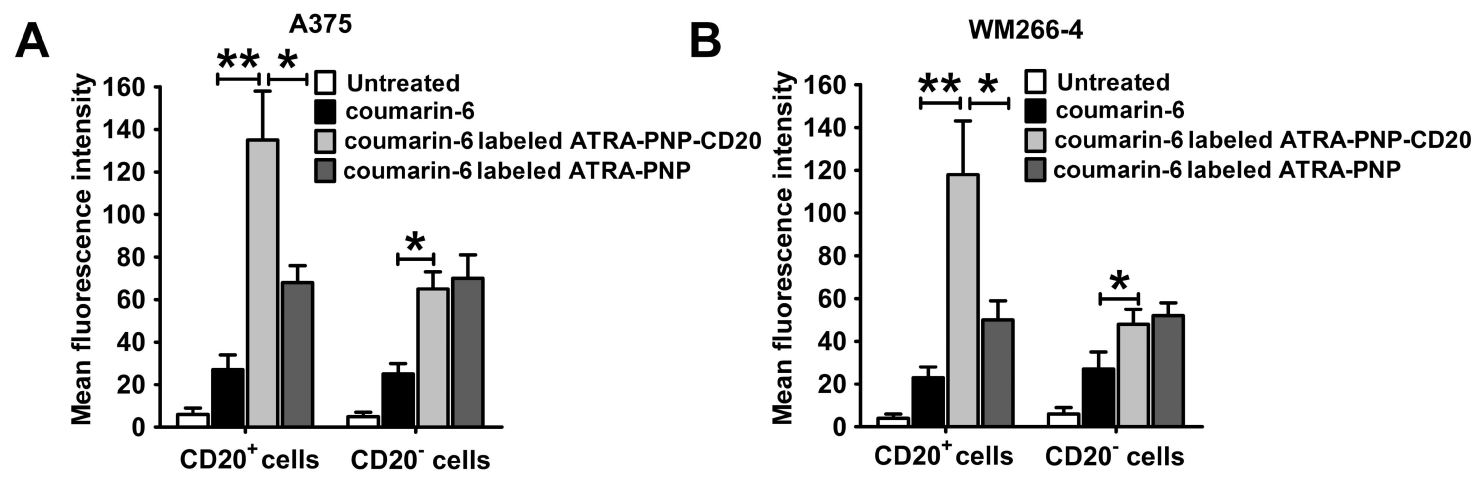

Figure 3 In vitro targeting of coumarin 6-labeled nanoparticles to melanoma cells.

Notes: (A) A375 cells and (B) WM266-4 cells. The difference between two groups was evaluated by one-way ANOVA with the Newman-Keuls posttest. $* P<0.05$; $* * P<0.01$. Data are presented as mean \pm SD $(n=3)$. ATRA-PNP, ATRA-encapsulated PLGA nanoparticles; ATRA-PNP-CD20, ATRA-encapsulated PLGA nanoparticles conjugated with CD20 antibodies; PNP-CD20, blank PLGA nanoparticles conjugated with CD20 antibodies.

Abbreviations: ATRA, all-trans retinoic acid; PLGA, poly(lactic-co-glycolic acid).

uptake of coumarin 6-labeled ATRA-PNP-CD20 and ATRAPNP, uptake was still higher than that of free coumarin 6 $(P<0.05)$. In the case of WM266-4 cells, similar results were achieved (Figure 3B). CD20+ WM266-4 cells showed a higher uptake of coumarin 6-labeled ATRA-PNP-CD20 than that of coumarin 6-labeled ATRA-PNP $(P<0.01)$ and free coumarin $6(P<0.05)$, whereas CD20- WM266-4 cells showed a similar uptake of coumarin 6-labeled ATRA-PNPCD20 and ATRA-PNP.

\section{Cytotoxic effects of ATRA and various nanoparticles against melanoma cells} Figure 4 shows that PNP-CD20, ie, blank PLGA nanoparticles with the CD20 antibody, displayed no remarkable
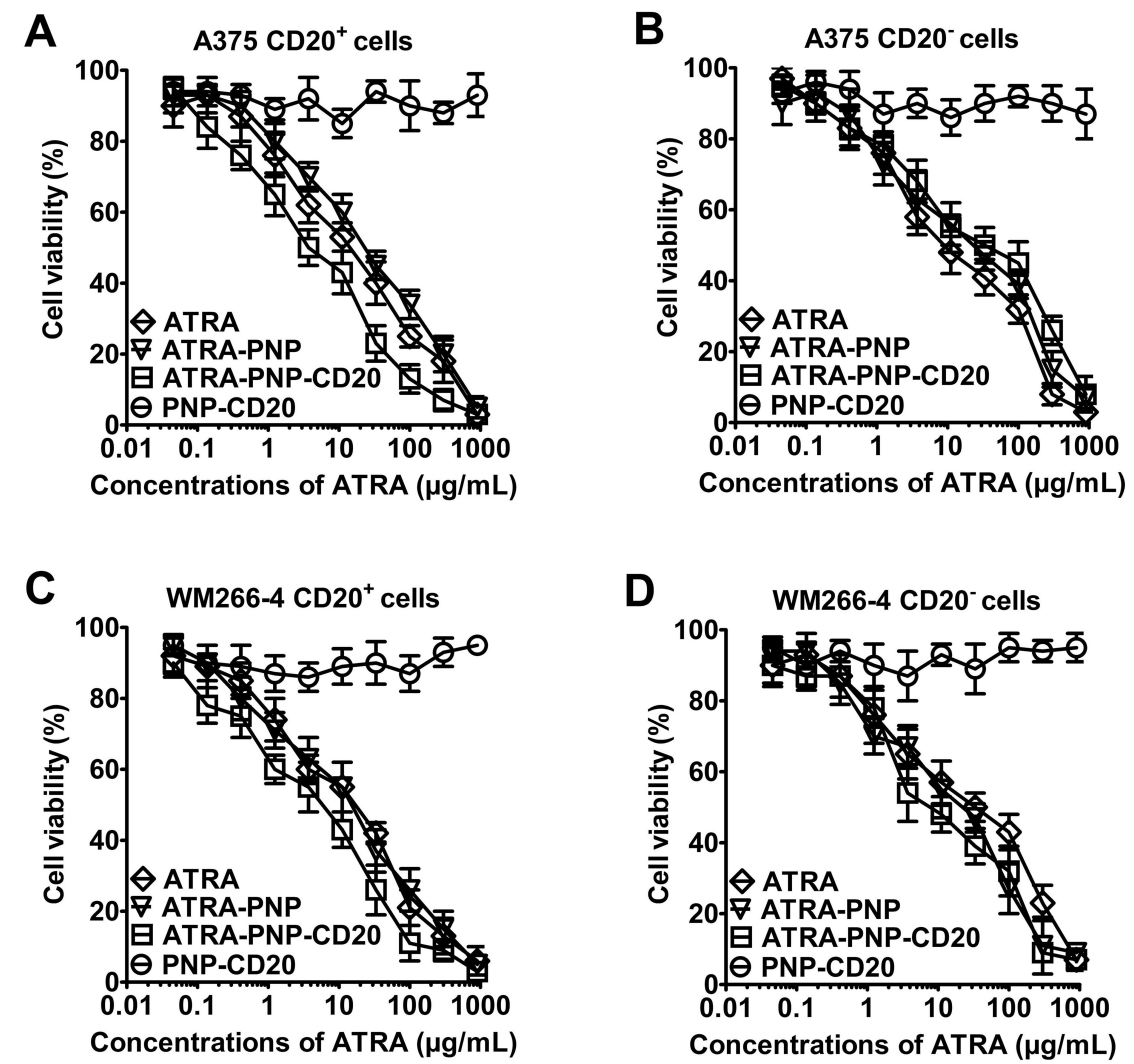

Figure 4 Cytotoxicity of nanoparticles for melanoma cells was examined using the CCK- 8 assay.

Notes: (A) A375 CD20+ cells, (B) A375 CD20- cells, (C) WM266-4 CD20+ cells, and (D) WM266-4 CD20- cells treated with nanoparticles. Data are presented as mean \pm SD $(n=3)$. ATRA-PNP, ATRA-encapsulated PLGA nanoparticles; ATRA-PNP-CD20, ATRA-encapsulated PLGA nanoparticles conjugated with CD20 antibodies; PNP-CD20, blank PLGA nanoparticles conjugated with CD20 antibodies.

Abbreviations: ATRA, all-trans retinoic acid; CCK-8, cell counting kit-8; PLGA, poly(lactic-co-glycolic acid). 
cytotoxic effects against melanoma cells. On the contrary, the dose-dependent cytotoxicity was observed for ATRA, ATRA-PNP, and ATRA-PNP-CD20. The $\mathrm{IC}_{50}$ values of ATRA and the nanoparticles are listed in Table 3. ATRAPNP and ATRA showed similar cytotoxic effects against CD20 ${ }^{+}$A375 cells ( IC $_{50}$ values: 23.6 and $19.8 \mu \mathrm{g} / \mathrm{mL}$, respectively); however, ATRA-PNP-CD20 showed a significantly higher cytotoxic effect $\left(\mathrm{IC}_{50}: 3.8 \mu \mathrm{g} / \mathrm{mL} ; P<0.001\right)$. Meanwhile, the $\mathrm{IC}_{50}$ values of ATRA-PNP-CD20 $(50.2 \mu \mathrm{g} / \mathrm{mL})$, ATRA-PNP $(52.1 \mu \mathrm{g} / \mathrm{mL})$, and ATRA $(48.3 \mu \mathrm{g} / \mathrm{mL})$ did not differ markedly against $\mathrm{CD} 20^{-} \mathrm{A} 375$ cells. In the case of WM266-4 cells, the cytotoxic effect of ATRA-PNP-CD20 was also remarkably higher than those of ATRA-PNP and ATRA $(P<0.01)$, whereas all three formulations showed similar cytotoxic effects for CD20- WM266-4 cells. In summary, ATRA-PNP-CD20 showed a preferential cytotoxic effect toward CD20+ melanoma cells.

\section{Impact of nanoparticles on the proportion of melanoma-initiating cells}

Figure 5 shows the impact of nanoparticles on the percentage of melanoma-initiating cells in the melanoma cell line populations. Treatment of A375 cells with ATRA remarkably inhibited the number of tumorspheres $(P<0.05$; Figure 5A). The inhibitory ability of ATRA was similar to that of ATRAPNP. Notably, the number of tumorspheres significantly decreased after ATRA-PNP-CD20 treatment compared with that after ATRA and ATRA-PNP treatments $(P<0.05)$. The treatment with PNP-CD20 had no effect on the number of tumorspheres. Similar results were achieved with WM266-4 cells. Although the number of tumorspheres was not affected by the treatment with PNP-CD20, ATRA-PNP-CD20 exhibited the best efficacy in inhibiting the number of tumorspheres formed by WM266-4 cells.

Table 3 The $I_{50}$ of ATRA and nanoparticles in melanoma cells at 72 hours

\begin{tabular}{l|l|l|l|l}
\hline \multirow{2}{*}{$\mathrm{IC}_{50}(\mu \mathrm{g} / \mathrm{mL})$} & \multicolumn{3}{|l|}{ A375 } & \multicolumn{2}{l}{ WM266-4 } \\
\cline { 2 - 5 } & $\mathbf{C D 2 0}^{+}$ & $\mathrm{CD20}^{-}$ & CD20 $^{+}$ & CD20- $^{-}$ \\
\hline ATRA & $19.8 \pm 3.6$ & $48.3 \pm 8.2$ & $25.5 \pm 6.8$ & $52.7 \pm 8.2$ \\
ATRA-PNP & $23.6 \pm 4.3$ & $52.1 \pm 8.1$ & $30.9 \pm 6.4$ & $67.6 \pm 7.3$ \\
ATRA-PNP-CD20 & $3.8 \pm 1.8$ & $50.2 \pm 8.5$ & $6.6 \pm 2.6$ & $62.5 \pm 6.5$ \\
PNP-CD20 & $>900.0$ & $>900.0$ & $>900.0$ & $>900.0$ \\
\hline
\end{tabular}

Notes: Data are expressed as mean \pm SD $(n=3)$. ATRA-PNP, ATRA-encapsulated PLGA nanoparticles; ATRA-PNP-CD20, ATRA-encapsulated PLGA nanoparticles conjugated with CD20 antibodies; PNP-CD20, blank PLGA nanoparticles conjugated with CD20 antibodies.

Abbreviations: ATRA, all-trans retinoic acid; $\mathrm{IC}_{50}$, half-maximal inhibitory concentration; PLGA, poly(lactic-co-glycolic acid).
Consistent with the abovementioned results, the percentage of $\mathrm{CD} 20^{+} \mathrm{A} 375$ cells was remarkably reduced after ATRA treatment ( $P<0.05$; Figure 5C). ATRA-PNP showed a similar inhibitory effect on the percentage of $\mathrm{CD} 20^{+} \mathrm{A} 375$ cells, while again, the percentage of CD20 ${ }^{+}$ A375 cells was the lowest among the groups after treatment with ATRA-PNP-CD20. As shown in Figure 5D, similar results were obtained in the case of WM266-4 cells.

Thus, ATRA-PNP-CD20 showed the best efficacy in the inhibition of tumorsphere formation and the percentage of $\mathrm{CD}_{20}{ }^{+}$cells in the melanoma cell population, thus representing a promising treatment for the elimination of melanomainitiating cells.

\section{Discussion}

Since melanoma-initiating cells are considered to be the dark seed of melanoma, their eradication may result in a better anticancer therapeutic effect. Considering that CD20 is the marker of melanoma-initiating cells, herein, we constructed ATRA-PNP-CD20, to target melanoma-initiating cells. In this study, ATRA-PNP-CD20 showed significantly better inhibitory effects against melanoma-initiating cells than did free ATRA and nontargeted nanoparticles.

ATRA is a promising drug that has shown potential therapeutic effects in various cancers, such as head and neck squamous cell carcinoma and bladder cancer. ${ }^{11}$ It has shown promising effects on the cell growth, differentiation, and apoptosis of cancer cells ${ }^{11,12}$ and a therapeutic potential against CICs in several cancers such as breast cancer. ${ }^{13-15}$ To the best of our knowledge, there have been no studies reporting a therapeutic effect of ATRA on melanomainitiating cells. In this study, we confirmed, using a cytotoxicity assay, that ATRA preferentially eliminated CD20 ${ }^{+}$ melanoma-initiating cells over $\mathrm{CD}^{2} 0^{-}$melanoma cells. In the tumorsphere formation assay, ATRA also reduced the number of tumorspheres formed by melanoma cells. Consistently, the percentage of $\mathrm{CD} 20^{+}$cells in the population of melanoma cells significantly decreased after ATRA treatment. To the best of our knowledge, this is the first report that demonstrated that ATRA could show potential activity toward melanoma-initiating cells.

Antibody-conjugated nanoparticles are a promising tool against various cancers because they can significantly enhance the therapeutic efficacy of chemotherapeutic drugs. ${ }^{32,33}$ Notably, three types of antibody-conjugated nanoparticles, loaded with doxorubicin or docetaxel, have been 
A

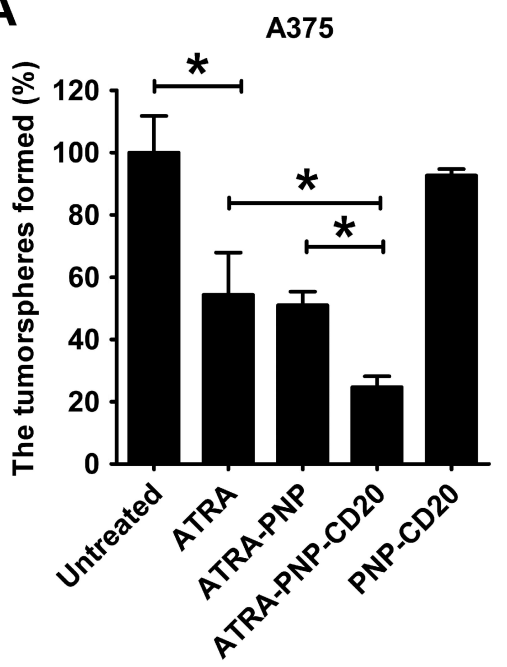

C

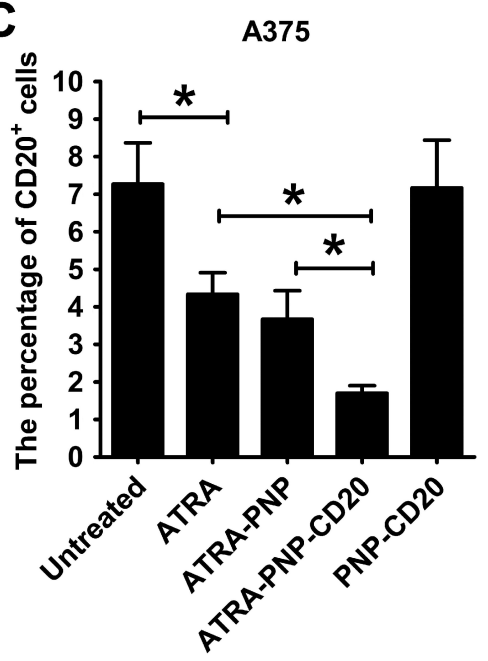

B

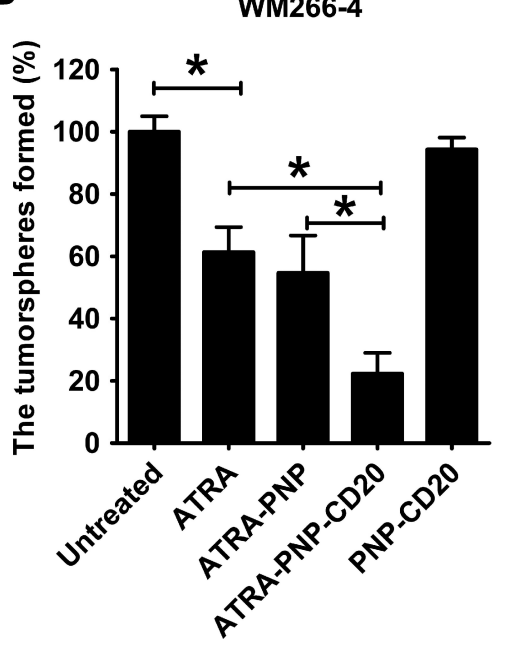

D

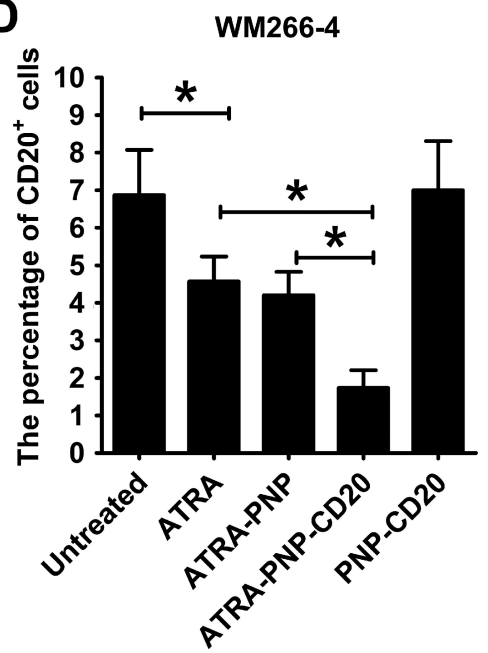

Figure 5 Impact of nanoparticles on the percentages of melanoma-initiating cells in the populations of A375 and WM266-4 melanoma cells, as determined by the evaluation of the tumorsphere formation (A and $\mathbf{B}$ ) and percentage of $\mathrm{CD} 20^{+}$cells ( $\mathbf{C}$ and $\mathbf{D}$ ).

Notes: The percentage of $\mathrm{CD}_{2} \mathrm{O}^{+}$cells was measured by flow cytometry after the treatment of melanoma cells. The difference between two groups was evaluated by one-way ANOVA with the Newman-Keuls posttest. $* P<0.05$. Data are presented as mean \pm SD $(n=3)$. ATRA-PNP, ATRA-encapsulated PLGA nanoparticles; ATRA-PNP-CD20, ATRA-encapsulated PLGA nanoparticles conjugated with CD20 antibodies; PNP-CD20, blank PLGA nanoparticles conjugated with CD20 antibodies.

Abbreviations: ATRA, all-trans retinoic acid; PLGA, poly(lactic-co-glycolic acid).

successfully translated into early-phase clinical trials, ${ }^{32,33}$ and the results demonstrated good safety and efficacy profiles of the antibody-conjugated nanoparticles. Tumor antigens are very important for selecting an antibody for cancer-targeting nanoparticles. CD20 is regarded as a good target for the treatment of melanoma-initiating cells. First, many normal tissues do not express CD20, and only a small number of tissues, such as B cells or their derived malignancies, express CD20. ${ }^{34}$ On the contrary, other markers of CICs, such as CD133 and CD44, are widely expressed in normal tissues. ${ }^{7}$ The limited expression of CD20 in normal tissues minimizes damage to normal tissues when targeting CD20. Second, CD20 is a stationary and accessible target, and it is not shed or secreted into blood circulation. ${ }^{35}$ Furthermore, the selection of a CD20 antibody was very critical for specific targeting of our developed nanoparticles to melanoma-initiating cells. The results showed that ATRA-PNP-CD20 significantly better targeted $\mathrm{CD}^{2} 0^{+}$melanoma-initiating cells than did ATRA-PNP, resulting in higher cytotoxic effects and inhibitory effects on tumorsphere formation. However, the cytotoxicity and tumorsphere inhibitory effects of ATRA-PNP-CD20 did not differ from those of ATRA-PNP in the case of CD20melanoma cells. These data firmly demonstrated that ATRAPNP-CD20 were able to better target and to exert stronger inhibitory effects against melanoma-initiating cells. To the best of our knowledge, this is the first report on targeted 
delivery of ATRA to melanoma-initiating cells via CD20 antibody-conjugated nanoparticles.

Unlike biodegradable organic nanoparticles, inorganic nanoparticles cannot be degraded and may cause damage to human beings. ${ }^{36,37}$ Therefore, the potential clinical use of inorganic nanoparticles is remarkably limited by their poor safety. ${ }^{36}$ On the contrary, biodegradable organic nanoparticles are more promising in clinical applications because of their superior safety. ${ }^{36,37}$ In our study, the components of the ATRAPNP-CD20 drug delivery system included PLGA and a CD20 antibody, which are safe according to US Food and Drug Administration (FDA)-approved materials. Regarding ATRA, FDA has recently approved the combination use of arsenic trioxide (Trisenox ${ }^{\circledR}$; Cell Therapeutics, Inc. Seattle, WA, USA.) injection plus ATRA as a first-line treatment for low-risk APL. In our study, PNP-CD20, ie, blank PLGA nanoparticles with the CD20 antibody, displayed no remarkable cytotoxic effects on melanoma cells. These preliminary safety data indicated that our nanoparticles represented a safe drug delivery system. Thus, ATRA-PNP-CD20 is expected to be safe in the clinic, which will facilitate their clinical translation.

\section{Conclusion}

This study is the first report of the anticancer activity of ATRA against melanoma-initiating cells and also the first report on the facilitation of drug delivery to melanoma-initiating cells via nanoparticles by using a CD20 antibody. ATRA-PNPCD20 nanoparticles were confirmed to selectively target $\mathrm{CD}^{2} 0^{+}$melanoma-initiating cells. Therefore, the use of ATRA-PNP-CD20 represents a promising approach for the therapy of melanoma-initiating cells. Since melanomainitiating cells are crucial for drug resistance and metastasis of melanoma, patients with melanoma may benefit from targeted therapy of melanoma-initiating cells.

\section{Acknowledgment}

This study was supported by the National Natural Science Foundation of China (81402512).

\section{Disclosure}

The authors report no conflicts of interest in this work.

\section{References}

1. Siegel RL, Miller KD, Jemal A. Cancer Statistics, 2017. CA Cancer J Clin. 2017;67(1):7-30.

2. Wong DJ, Ribas A. Targeted therapy for melanoma. Cancer Treat Res. 2016;167:251-262.

3. Gray-Schopfer V, Wellbrock C, Marais R. Melanoma biology and new targeted therapy. Nature. 2007;445(7130):851-857.
4. Maverakis E, Cornelius LA, Bowen GM, et al. Metastatic melanoma-a review of current and future treatment options. Acta Derm Venereol. 2015;95(5):516-524.

5. Zabierowski SE, Herlyn M. Melanoma stem cells: the dark seed of melanoma. J Clin Oncol. 2008;26(17):2890-2894.

6. Roesch A. Cells Mstem. Melanoma stem cells. J Dtsch Dermatol Ges. 2015;13(2):118-124.

7. Gao J, Li W, Guo Y, Feng SS. Nanomedicine strategies for sustained, controlled and targeted treatment of cancer stem cells. Nanomedicine. 2016;11(24):3261-3282.

8. Fang D, Nguyen TK, Leishear K, et al. A tumorigenic subpopulation with stem cell properties in melanomas. Cancer Res. 2005;65(20): 9328-9337.

9. Schmidt P, Kopecky C, Hombach A, Zigrino P, Mauch C, Abken H. Eradication of melanomas by targeted elimination of a minor subset of tumor cells. Proc Natl Acad Sci U S A. 2011;108(6):2474-2479.

10. Pinc A, Somasundaram R, Wagner C, et al. Targeting CD20 in melanoma patients at high risk of disease recurrence. Mol Ther. 2012; 20(5):1056-1062.

11. Siddikuzzaman, Guruvayoorappan C, Berlin Grace VM. All trans retinoic acid and cancer. Immunopharmacol Immunotoxicol. 2011;33(2): 241-249.

12. Montesinos P, Bergua JM, Vellenga E, et al. Differentiation syndrome in patients with acute promyelocytic leukemia treated with all-trans retinoic acid and anthracycline chemotherapy: characteristics, outcome, and prognostic factors. Blood. 2009;113(4):775-783.

13. Li RJ, Ying X, Zhang Y, et al. All-trans retinoic acid stealth liposomes prevent the relapse of breast cancer arising from the cancer stem cells. J Control Release. 2011;149(3):281-291.

14. Karsy M, Albert L, Tobias ME, Murali R, Jhanwar-Uniyal M. Alltrans retinoic acid modulates cancer stem cells of glioblastoma multiforme in an MAPK-dependent manner. Anticancer Res. 2010;30(12): 4915-4920.

15. Han D, Rodriguez-Bravo V, Charytonowicz E, et al. Targeting sarcoma tumor-initiating cells through differentiation therapy. Stem Cell Res. 2017:21:117-123.

16. Zhao X, Graves C, Ames SJ, Fisher DE, Spanjaard RA. Mechanism of regulation and suppression of melanoma invasiveness by novel retinoic acid receptor-gamma target gene carbohydrate sulfotransferase 10 . Cancer Res. 2009;69(12):5218-5225.

17. Zhang H, Satyamoorthy K, Herlyn M, Rosdahl I. All-trans retinoic acid (atRA) differentially induces apoptosis in matched primary and metastatic melanoma cells - a speculation on damage effect of atRA via mitochondrial dysfunction and cell cycle redistribution. Carcinogenesis. 2003;24(2):185-191.

18. Ishibashi M, Arai M, Tanaka S, Onda K, Hirano T. Antiproliferative and apoptosis-inducing effects of lipophilic vitamins on human melanoma A375 cells in vitro. Biol Pharm Bull. 2012;35(1):10-17.

19. Chen X, Yang M, Hao W, et al. Differentiation-inducing and antiproliferative activities of isoliquiritigenin and all-trans-retinoic acid on B16F0 melanoma cells: Mechanisms profiling by RNA-seq. Gene. 2016; 592(1):86-98.

20. Yin W, Song Y, Liu Q, Wu Y, He R. Topical treatment of all-trans retinoic acid inhibits murine melanoma partly by promoting $\mathrm{CD} 8^{+}$ T-cell immunity. Immunology. 2017;152(2):287-297.

21. Chen D, Xie F, Sun D, Yin C, Gao J, Zhong Y. NanomedicineMediated Combination Drug Therapy in Tumor. Open Pharm Sci J. 2017;4(1):1-10

22. Xie FY, Xu WH, Yin C, Zhang GQ, Zhong YQ, Gao J. Nanomedicine strategies for sustained, controlled, and targeted treatment of cancer stem cells of the digestive system. World J Gastrointest Oncol. 2016; 8(10):735-744.

23. Gao J, Feng SS, Guo Y. Nanomedicine against multidrug resistance in cancer treatment. Nanomedicine. 2012;7(4):465-468.

24. Cristiano MC, Cosco D, Celia C, et al. Anticancer activity of all-trans retinoic acid-loaded liposomes on human thyroid carcinoma cells. Colloids Surf B Biointerfaces. 2017;150:408-416. 
25. Feng SS. Nanoparticles of biodegradable polymers for new-concept chemotherapy. Expert Rev Med Devices. 2004;1(1):115-125.

26. Gao J, Xia Y, Chen H, et al. Polymer-lipid hybrid nanoparticles conjugated with anti-EGF receptor antibody for targeted drug delivery to hepatocellular carcinoma. Nanomedicine. 2014;9(2):279-293.

27. Gao J, Chen H, Song H, et al. Antibody-targeted immunoliposomes for cancer treatment. Mini Rev Med Chem. 2013;13(14):2026-2035.

28. Gao J, Feng SS, Guo Y. Antibody engineering promotes nanomedicine for cancer treatment. Nanomedicine. 2010;5(8):1141-1145.

29. Wang J, Wu Z, Pan G, et al. Enhanced doxorubicin delivery to hepatocellular carcinoma cells via CD147 antibody-conjugated immunoliposomes. Nanomedicine. 2018;14(6):1949-1961.

30. Song H, Su X, Yang K, et al. CD20 Antibody-Conjugated Immunoliposomes for Targeted Chemotherapy of Melanoma Cancer Initiating Cells. J Biomed Nanotechnol. 2015;11(11):1927-1946.

31. Zeng YB, Yu ZC, He YN, et al. Salinomycin-loaded lipid-polymer nanoparticles with anti-CD20 aptamers selectively suppress human CD20+ melanoma stem cells. Acta Pharmacol Sin. 2018;39(2):261-274.

32. Mamot C, Ritschard R, Wicki A, et al. Tolerability, safety, pharmacokinetics, and efficacy of doxorubicin-loaded anti-EGFR immunoliposomes in advanced solid tumours: a phase 1 dose-escalation study. Lancet Oncol. 2012;13(12):1234-1241.
33. Miller K, Cortes J, Hurvitz SA, et al. HERMIONE: a randomized Phase 2 trial of MM-302 plus trastuzumab versus chemotherapy of physician's choice plus trastuzumab in patients with previously treated, anthracycline-naïve, HER2-positive, locally advanced/metastatic breast cancer. BMC Cancer. 2016;16:352.

34. Maloney DG. Anti-CD20 antibody therapy for B-cell lymphomas. N Engl J Med. 2012;366(21):2008-2016.

35. Beers SA, Chan CH, French RR, Cragg MS, Glennie MJ. CD20 as a target for therapeutic type I and II monoclonal antibodies. Semin Hematol. 2010;47(2):107-114.

36. Auffan M, Rose J, Bottero JY, Lowry GV, Jolivet JP, Wiesner MR. Towards a definition of inorganic nanoparticles from an environmental, health and safety perspective. Nat Nanotechnol. 2009;4(10):634-641.

37. Cushing BL, Kolesnichenko VL, O'Connor CJ. Recent advances in the liquid-phase syntheses of inorganic nanoparticles. Chem Rev. 2004;104(9):3893-3946.
OncoTargets and Therapy

\section{Publish your work in this journal}

OncoTargets and Therapy is an international, peer-reviewed, open access journal focusing on the pathological basis of all cancers, potential targets for therapy and treatment protocols employed to improve the management of cancer patients. The journal also focuses on the impact of management programs and new therapeutic agents and protocols on

\section{Dovepress}

patient perspectives such as quality of life, adherence and satisfaction. The manuscript management system is completely online and includes a very quick and fair peer-review system, which is all easy to use. Visit http://www.dovepress.com/testimonials.php to read real quotes from published authors. 